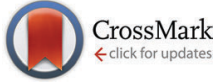

Cite this: Phys. Chem. Chem. Phys., 2015, 17, 15579

Received 15th December 2014 Accepted 2nd March 2015

DOI: $10.1039 / c 4 c p 05872 k$

www.rsc.org/pccp

\title{
Release of proteins and enzymes from vesicular compartments by alternating magnetic fields $\dagger$
}

\author{
Andrew Booth, ${ }^{a b c}$ Inmaculada C. Pintre, ${ }^{a b}$ Yue Lin, ${ }^{c}$ Julie E. Gough*c and \\ Simon J. Webb*ab
}

\begin{abstract}
The magnetic release of catalytically active enzymes from vesicular compartments within aggregated nanomaterials has been demonstrated. These nanomaterials, magnetic nanoparticle-vesicle aggregates (MNPVs), were formed by the self-assembly of biotinylated silica-coated $\mathrm{Fe}_{3} \mathrm{O}_{4}$ nanoparticles, biotinylated vesicles and tetrameric avidin. The unique features of nanoscale magnetite allow adhesion between membranes to be combined with magnetically triggered transit of reagents across membranes. Adding short spacers between the adhesive biotin groups and the nanoparticle or vesicle surfaces was found to strengthen binding to avidin, with binding of avidin to biotinylated bilayers and biotinylated nanoparticles monitored by quartz crystal microgravimetry with dissipation (QCM-D). Three different reagents were released from the vesicle compartments of MNPVs by a pulse of alternating magnetic field, with the release of a dye modelling the release of small molecule substrates, and the release of cytochrome $c$ modelling the release of biological polymers, such as enzymes. To confirm that enzymes could be released and maintain activity, trypsin was encapsulated and shown to digest casein after magnetically triggered release.
\end{abstract}

\section{Introduction}

Phospholipid bilayers play a crucial role in vivo by delineating compartments within cells or in tissue. Within tissue, these compartments - individual cells - have distinctive environments containing biochemical networks separated in space but communicating through pores or receptors in the membranes. These networks in vivo use a complex mixture of catalysis and feedback loops to regulate compound flux through the network pathways, notwithstanding any transmembrane communication between networks in different compartments. Since increasing the distance between compartments diminishes the fidelity of communication, adhesion between cells facilitates information transfer. Indeed these functions of adhesion and communication are combined in gap junctions, which are adhesive channels that directly connect the cytoplasm of different cells and facilitate rapid information (chemical) transfer. However, rather than

\footnotetext{
${ }^{a}$ Manchester Institute of Biotechnology, University of Manchester,

131 Princess Street, Manchester, M1 7DN, UK. E-mail: S.Webb@manchester.ac.uk

${ }^{b}$ School of Chemistry, University of Manchester, Oxford Road, Manchester, M13 9PL, UK

${ }^{c}$ School of Materials, University of Manchester, Grosvenor St, Manchester, M13 9PL, UK. E-mail:J.Gough@manchester.ac.uk

$\dagger$ Electronic supplementary information (ESI) available: FTIR spectra, surface functionalisation assays and SAR measurements on MNPs. Additional QCM-D traces. Microscopy images of MNPVs. Full experimental details of MNPV synthesis and magnetic release experiments. See DOI: 10.1039/c4cp05872k
}

being non-selective pores, these gap junctions have been shown to exhibit selectivity over the compounds allowed to pass between cells. ${ }^{1}$

Creating "gates" between bilayer defined compartments that can be remotely opened would be a first step towards biomimetic systems able to produce a better understanding of how biochemical networks in tissue communicate. ${ }^{2}$ There are several elegant methods for bringing compartments into proximity and allowing inter-compartment communication, including oil-in-water droplets and giant unilamellar vesicles (GUVs) that communicate through $\alpha$-hemolysin channels, ${ }^{3,4}$ as well as GUVs linked by phospholipid tubules. ${ }^{5}$ However these techniques do not involve the self-assembly of populations of vesicles, instead requiring either micromechanical/microfluidic manipulation or printing of individual compartments.

Creating vesicle-based systems able to self-assemble into bulk materials that combine membrane adhesion and communication is an ongoing challenge. ${ }^{6}$ Several supramolecular interactions have been developed to mediate vesicle self-assembly, from the relatively weak, such as histidine coordination to copper(II) centres, ${ }^{7}$ to the relatively strong, like avidin-mediated crosslinking of biotinylated vesicles. ${ }^{8}$ Other motifs have included DNA hybridisation, ${ }^{9}$ peptide-peptide interactions ${ }^{10}$ and phosphateguanidinium interactions. ${ }^{11}$ Multivalency across the interacting surfaces is thought to be important, as is clustering of adhesive lipids in the membrane. ${ }^{12}$ Beyond providing a better understanding of communication between cells in tissue, creating biomimetic 
a)

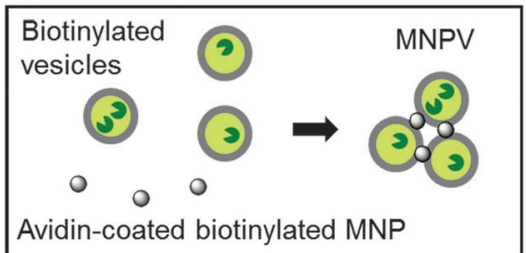

b)

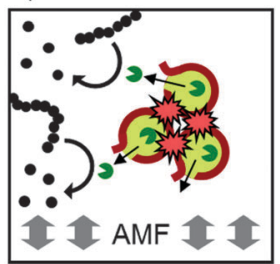

Fig. 1 (a) Formation of MNPVs from avidin-coated biotinylated magnetic nanoparticles (MNPs) and biotinylated phospholipid vesicles. (b) Release of encapsulated enzymes in response to an alternating magnetic field (AMF).

self-assembled systems able to combine membrane adhesion with chemical communication could produce new technology with diverse applications in drug delivery and smart biomaterials, potentially allowing the delivery of biochemical agents directly to the interior of targeted cells.

Our self-assembly approach to combining adhesion with transport has used adhesive magnetic nanoparticles to crosslink large unilamellar phospholipid vesicles (LUVs), creating a new bionanotechnological drug delivery system; magnetic nanoparticle-vesicle assemblies (MNPVs, Fig. 1) ${ }^{13}$ MNPVs consist of LUVs with stored compounds that are crosslinked by $\mathrm{Fe}_{3} \mathrm{O}_{4}$ magnetic nanoparticles, creating a nanomaterial with vesicle compartments. ${ }^{14}$ The unique properties of nanosized $\mathrm{Fe}_{3} \mathrm{O}_{4}$ fulfill two critical roles: (a) they allow magnetic separation of MNPVs and objects linked to them; ${ }^{14}$ (b) they allow nondestructive release of vesicle contents by an alternating magnetic field (AMF). ${ }^{15}$ The latter effect has been ascribed to AMF-induced localised heating of the MNPs, which "melts" the vesicle bilayers around the MNPs ${ }^{13,16}$ and allows compounds to pass through the bilayer at boundaries between coexisting solid ordered $\left(\mathrm{s}_{\mathrm{o}}\right)$ and liquid ordered $\left(l_{d}\right)$ phases. ${ }^{6}$

As most cells are unaffected by oscillating or permanent magnetic fields, such remote control over release has potential applications both in vitro and in vivo. For example chondrocytes were unaffected either by an AMF or by MNPVs storing ascorbate2-phosphate (AAP) separately. However the combination of both released the AAP from the MNPVs and induced a cellular response, collagen production by chondrocytes, which added this natural extracellular matrix to the synthetic alginate hydrogel. ${ }^{15 b}$ Magnetic patterning of MNPVs in a hydrogel matrix has also been used to give spatial control over cellular responses to released compounds. ${ }^{15 a}$

Extending compound release from MNPVs, which is currently limited to small molecules like dyes and ions, to the release of substrates and enzymes would be a first step towards a biomimetic self-assembled system with remotely controllable gates between biochemical networks. However several barriers need to be overcome, including robust methods for enzyme encapsulation and showing magnetic release from MNPVs in suspension; our previous examples of magnetic release have been from MNPVs immobilised in hydrogel matrices. ${ }^{15}$ Reported herein are studies of the encapsulation and magnetic release of proteins from a redesigned MNPV system in suspension.

\section{Experimental procedures}

\section{Instrumentation}

Fluorescence measurements were recorded using a Perkin Elmer LS55 fluorimeter, UV-visible spectrophotometry was performed on a V-660 spectrometer (Jasco (UK) Ltd). Vesicles were extruded using a LiposoFast liposome extruder (AVESTIN Europe GmbH). Sonication was performed using a VCX130PB probe-type sonicator (Sonics \& Materials Inc., $130 \mathrm{~W}$ power rating). Centrifugation was performed using a Heraeus Instruments Megafuge 1.0R for large tubes and a Beckman Coulter Microfuge 16 for Eppendorf tubes. Vortex mixing was performed using a Vortex Genie 2 (600-2700 rpm). Transmission electron microscopy (TEM) was performed using a Jeol 1220, $120 \mathrm{kV}$ instrument with a GATAN ORIUS CCD camera or a Tecnai T20, $220 \mathrm{kV}$. Inductive heating of nanoparticles was conducted using a water-cooled EASYHEAT 0224 induction heater $(2.0 \mathrm{~kW}, 150-400 \mathrm{kHz})$ with an EASYHEAT 300P workhead $(392 \mathrm{kHz}, 3 \mathrm{~cm}$ loop diameter, 1.5 turns). QCM-D was performed using a Q-Sense E4 instrument (Biolin Scientific, UK). NdFeB magnets ring/cylinder shape (5600 Gauss) were obtained from Magnet Expert Ltd (UK).

\section{Materials}

Dipalmitoyl phosphatidylcholine (DPPC), dioleoyl phosphatidylcholine (DOPC) and biotinylated lipids were obtained from Avanti Polar Lipids Inc., (AL, USA). Avidin was obtained from Invitrogen (Thermo Fisher Scientific Inc. (USA)). Unless stated otherwise, all other regents were obtained from Sigma-Aldrich (UK). Polycarbonate membranes ( $800 \mathrm{~nm}$ pore diameter) for vesicle extrusion were obtained from AVESTIN Europe GmbH. PD-10 Sephadex gel-permeation desalting columns were obtained from GE Healthcare Bio-Sciences AB (Sweden).

\section{APTES nanoparticle coating procedure}

Uncoated $\mathrm{Fe}_{3} \mathrm{O}_{4}$ MNPs (Sigma-Aldrich Co. UK, Product No. $637106,20 \mathrm{mg}, 87 \mu \mathrm{mol}$ ) in a $50 \mathrm{~mL}$ plastic tube were suspended in $\mathrm{MeOH}(5 \mathrm{~mL})$ using a probe sonicator until a homogeneous suspension was obtained ( $\sim 20$ minutes). To this suspension was added APTES $(0.177 \mathrm{~g}, 0.187 \mathrm{~mL}, 800 \mu \mathrm{mol})$, followed by sonication for a further $1 \mathrm{~h}$. The sample tube was then sealed and the mixture was shaken overnight. The MNPs were sedimented using an $\mathrm{NdFeB}$ permanent magnet $(5600 \mathrm{G}$ ) on the exterior of the tube and the supernatant solution carefully decanted. The MNPs were then resuspended in fresh $\mathrm{MeOH}(5 \mathrm{~mL})$ using brief bath-type sonication, followed by sedimentation and removal of the supernatant. This process was then repeated at least 6 times. After the final resuspension, the suspension was transferred to a roundbottomed flask and the solvent was removed under reduced pressure, giving APTES-MNPs as a black to dark brown powder. In the acid-catalysed coating procedure, two to three drops of glacial acetic acid were added to the uncoated MNP suspension immediately prior to the addition of APTES.

Functionalisation of APTES-MNPs with 21-[D-(+)-biotinylamino]4,7,10,13,16,19-hexaoxaheneicosanoic acid (Bt-PEG-acid)

APTES-MNPs $(10 \mathrm{mg})$ were resuspended in dry $N, N$-dimethylformamide (DMF, $2 \mathrm{~mL}$ ) using probe sonication for 10 minutes. 
Bt-PEG-acid (5 mg, $3.45 \mu \mathrm{mol})$ and HBTU $(1.3 \mathrm{mg}, 3.45 \mu \mathrm{mol})$ were dissolved in dry DMF $(0.5 \mathrm{~mL})$ with $N, N$-diisopropylethylamine $(0.45 \mathrm{mg}, 3.45 \mu \mathrm{mol})$, and the mixture stirred for $1 \mathrm{~h}$ before being added to the MNP suspension. The resulting suspension was then sonicated for a further $1 \mathrm{~h}$, before being sealed in a plastic centrifuge tube $(15 \mathrm{~mL})$ and shaken overnight. Six rounds of magnetic sedimentation and resuspension in fresh DMF were performed, followed by removal of the solvent under reduced pressure. The Bt-APTES coated MNPs were obtained as a black solid.

\section{Production of phospholipid vesicles}

For small molecules like 5/6-carboxyfluorescein (5/6-CF), standard procedures for LUV production were followed using extrusion through $800 \mathrm{~nm}$ polycarbonate membranes (full details in the $\mathrm{ESI} \dagger$ ). For proteins, a freeze-thaw procedure was added to the standard vesicle preparation method. A lipid film was prepared in a $5 \mathrm{~mL}$ round-bottomed flask and resuspended in a solution of the desired protein (cytochrome $c$ or trypsin, $10 \mathrm{mg} \mathrm{mL}^{-1}$ in PBS). The resulting suspension was frozen in liquid nitrogen, then gently warmed evenly over the surface of the flask to thaw the suspension. After repeating this process 10 times, the vesicles were then extruded as described in the ESI. $\dagger$

Unencapsulated 5/6-CF was removed from vesicle suspensions ( $1 \mathrm{~mL}$ ) by gel permeation chromatography (GPC), using Sephadex G-25 PD-10 columns (exclusion limit $7 \mathrm{kDa}$ ). After GPC purification, the eluted suspension was returned to the original lipid concentration $(20 \mathrm{mM})$ by centrifugation at $2200 \times g$ for 15 minutes, followed by removal of the supernatant and resuspension in a total volume of $1 \mathrm{~mL}$ PBS. This procedure was then repeated.

For protein-loaded vesicles, after the freeze-thaw cycles and vesicle extrusion, the resulting suspension was sedimented in a centrifuge at $2200 \times g$ for 15 minutes or until the vesicles had sedimented to a $\sim 300 \mu \mathrm{L}$ volume. The supernatant was then removed and replaced with an equal volume of PBS. This process was repeated as many times as necessary to reduce the concentration of unencapsulated protein to below the detection limit of the technique being used to measure its release.

\section{Formation of MNPVs}

A suspension of Bt-APTES MNPs ( $2 \mathrm{~mL}, 0.5 \mathrm{mg} \mathrm{mL}{ }^{-1}$ in PBS) was prepared by probe sonication (set to $50 \%$ amplitude) for $\sim 10$ minutes. Care was taken to ensure the sample was not heated significantly by sonication; if observed the sample was allowed to cool to room temperature before continuing. Avidin ( $50 \mu \mathrm{L}$ of a $10 \mathrm{mg} \mathrm{mL}^{-1}$ solution in distilled water) was added to the MNP suspension, followed by brief vortex mixing and incubation for 5 minutes. Biotinylated vesicles $(200 \mu \mathrm{L}, 0.2 \mathrm{~mol} \%$ Bt-caproyl-PE in DPPC, $20 \mathrm{mM}$ total [lipid]) in PBS were then added to the avidin-MNP mixture followed by brief vortex mixing. The samples were then incubated at $37{ }^{\circ} \mathrm{C}$ for at least 40 minutes until aggregation was complete. Once the MNPVs had sedimented, the supernatant was removed and replaced with fresh PBS to give a total volume of $2 \mathrm{~mL}$.

\section{General procedure for magnetic release from MNPVs}

MNPV samples, prepared as above with a sedimented MNPV volume of $c a .300 \mu \mathrm{L}$, were placed in a $15 \mathrm{~mL}$ centrifuge tube and were either: (i) incubated at $42{ }^{\circ} \mathrm{C}$ (no AMF); (ii) incubated at $37^{\circ} \mathrm{C}$ and exposed to an AMF pulse (392 kHz, 5 minutes); (iii) incubated at $37{ }^{\circ} \mathrm{C}$ and not exposed to an AMF pulse.

For 5/6-CF release, aliquots of the supernatant $(20 \mu \mathrm{L})$ were removed at the desired timepoints, added to PBS $(2 \mathrm{~mL})$ and the fluorescence emission measured. After $24 \mathrm{~h}$, the samples were all heated to $42{ }^{\circ} \mathrm{C}$ for $15 \mathrm{~h}$ and then briefly sonicated $(\sim 120 \mathrm{~s})$ with a probe sonicator. An aliquot $(20 \mu \mathrm{L})$ was then taken, added to PBS $(2 \mathrm{~mL})$ and the fluorescence emission measured. Percentage release values were calculated relative to the fluorescence values obtained for these solutions.

For cytochrome $c$ release, at the desired timepoints the samples were sedimented by centrifugation at $2200 \times g$ and the absorbance spectrum of the supernatant recorded $\left(\lambda_{\max }=410 \mathrm{~nm}\right)$. The supernatant was returned to the MNPVs, which were resuspended and incubated at $37{ }^{\circ} \mathrm{C}$ between timepoints. Once the timecourse had been measured, the samples were sonicated ( $\sim 120 \mathrm{~s})$ using a probe sonicator and incubated at $42{ }^{\circ} \mathrm{C}$ for $1 \mathrm{~h}$. Percentage release values were calculated relative to the absorbance values obtained for these solutions.

For FITC-trypsin release, at the desired timepoints the samples were sedimented by centrifugation at $2200 \times g$, and the fluorescence emission of the supernatant $\left(\lambda_{\mathrm{ex}}=492 \mathrm{~nm}\right.$; $\lambda_{\mathrm{em}}=517 \mathrm{~nm}$ ) measured. The supernatant was returned to the MNPVs, which were resuspended and incubated at $37^{\circ} \mathrm{C}$ between timepoints. The concentration of released FITC-trypsin was calculated by interpolation of the fluorescence into a standard curve (see $\mathrm{ESI} \dagger$ for details).

For trypsin activity, the assay was performed on aliquots $(20 \mu \mathrm{L})$ of the supernatant buffer of the MNPVs taken at the desired timepoints. For each sample, incubation buffer $(20 \mu \mathrm{L}$, $20 \mathrm{mM}$ phosphate, $150 \mathrm{mM} \mathrm{NaCl}, \mathrm{pH}$ 7.6), FITC-casein solution $(20 \mu \mathrm{L})$ and the solution to be assessed for protease activity (MNPV supernatant or samples of standard trypsin solution, $10 \mu \mathrm{L}$ ) were combined in an Eppendorf tube. A blank was also prepared by combining incubation buffer $(20 \mu \mathrm{L})$, FITC-casein solution $(20 \mu \mathrm{L})$ and MilliQ water $(10 \mu \mathrm{L})$. All samples were vortex mixed and incubated for $1 \mathrm{~h}$ at $37^{\circ} \mathrm{C}$ in darkness. Trichloroacetic acid solution $(0.6 \mathrm{M}, 150 \mu \mathrm{L})$ was then added to each sample, vortex mixed and incubated in the dark for $0.5 \mathrm{~h}$. The tubes were then centrifuged at $10000 \times g$ for 10 minutes. Aliquots of the supernatant $(10 \mu \mathrm{L})$ were added to assay buffer $(1 \mathrm{~mL}$, Tris $500 \mathrm{mM}, \mathrm{pH} 8.5)$ and the fluorescence intensity of the solution was recorded at $535 \mathrm{~nm}\left(\lambda_{\mathrm{ex}}=485 \mathrm{~nm}\right)$. The fluorescence intensities were interpolated into a calibration plot established for samples of known trypsin activity (see the ESI $\dagger$ ).

\section{Results and discussion}

\section{APTES coating of $\mathrm{Fe}_{3} \mathrm{O}_{4}$ MNPs}

In previous work, a biotin-catechol conjugate was found to biotinylate the surface of synthesised MNPs with good efficiency. ${ }^{15 b}$ 
However to develop a more accessible protocol, a method of biotinylation was sought that would not require the synthesis of this biotin-catechol conjugate. Instead the popular silanation reagent (3-aminopropyl)triethoxysilane (APTES) was used to coat commercially available magnetite nanoparticles, ${ }^{17}$ resulting in a silica layer bearing pendant primary amine groups that allow further functionalization of the MNP surface using peptidecoupling chemistry. This stepwise method of functionalising MNP surfaces also allows each coating on the MNPs to be analysed and quantified.

Commercially available magnetite nanoparticles (uncoated $\mathrm{Fe}_{3} \mathrm{O}_{4}$ from Sigma-Aldrich, Product 637106, batch size $<50 \mathrm{~nm}$ ) were shown by dynamic light scattering to have a bimodal Gaussian size distribution with maxima at 36 and $55 \mathrm{~nm} .^{18}$ Measurement of the specific absorption rate (SAR) of these MNPs showed they were as effective as our synthesized MNP at converting magnetic energy into heat, with an increase of $44{ }^{\circ} \mathrm{C}$ at a concentration of $2.5 \mathrm{mg} \mathrm{mL}^{-1}$ during a $300 \mathrm{~s}$ AMF pulse (details in $\mathrm{ESI} \dagger$ ). These commercial MNPs were agitated in methanol using a probe sonicator to give a homogeneous suspension. APTES was added and the suspension was again sonicated, before being shaken overnight in a sealed centrifuge tube. In some cases a catalytic amount of acetic acid was added immediately after the APTES, as some reports have indicated that acid promotes APTES condensation and improves coating efficiencies. ${ }^{17 a}$ The MNPs were purified from non-magnetic material by repeated sedimentation using an NdFeB permanent magnet (5600 Gauss), removal and replacement of the supernatant with fresh methanol, and resuspension of the MNPs by sonication (six repeats). After the final resuspension, removal of the solvent under reduced pressure gave APTES-MNPS as a dark brown powder.

To characterise the APTES coatings on the MNPs, high resolution transmission electron microscopy (HRTEM) was performed on MNP samples both before and after APTES coating (Fig. 2).

For particles treated with APTES, an amorphous non-crystalline material both coated individual particles (Fig. 2(iv)) and bridged between MNPs (Fig. 2(iii)). This amorphous material is suggested to be silica deposited during the APTES coating procedure, forming a silica 'shell' around the $\mathrm{Fe}_{3} \mathrm{O}_{4}$ core. Although not measured across the whole sample, the TEM images suggested the average particle diameter of these APTES coated particles was 30-50 nm, similar to the diameter of the supplied MNPs $(<50 \mathrm{~nm})$ although there is also a greater degree of polydispersity of size and shape.

To confirm the core of these particles was $\mathrm{Fe}_{3} \mathrm{O}_{4}$, the lattice $d$ spacings were measured using Fourier transform of TEM images (e.g. Fig. 2(ii)). These were compared to the values for $\mathrm{Fe}_{3} \mathrm{O}_{4}$ reported by Sun et al. (Table 1) and were found to be a good match. ${ }^{19}$

A modification of the 2,4,6-trinitrobenzenesulfonic acid $(\mathrm{TNBS})^{20}$ colorimetric assay developed by Edwards-Lévy et al. was used to quantify the amount of amine available for reaction on the surface of these APTES MNPs. ${ }^{21}$ This modification of the traditional TNBS assay, termed a 'reverse' TNBS assay, is required to quantify amino groups on the surface of particles that generate too much turbidity in suspension to measure the absorption of
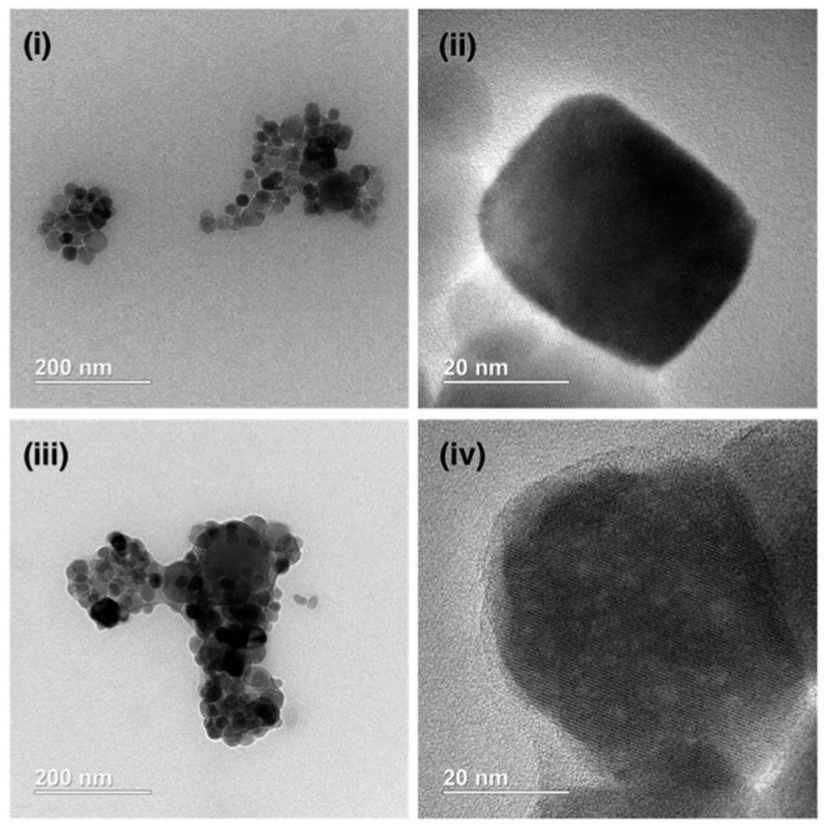

Fig. 2 Representative HRTEM images of ((i) and (ii)) uncoated $\mathrm{Fe}_{3} \mathrm{O}_{4}$ MNPs and ((iii) and (iv)) APTES coated commercial $\mathrm{Fe}_{3} \mathrm{O}_{4}$ MNPs.

Table 1 Measured $d$ spacings for the HRTEM Fourier transform of the image in Fig. 2(ii) compared to the known lattice spacings for $\mathrm{Fe}_{3} \mathrm{O}_{4}$ given by Sun et al., ${ }^{19}$ the corresponding $h k l$ (Miller) index is given for each $d$ spacing value

\begin{tabular}{lllllll}
\hline Measured $d$ spacings/ $/ \AA$ & 4.87 & 2.98 & 2.53 & 2.13 & 1.705 & 1.47 \\
$d$ spacings for $\mathrm{Fe}_{3} \mathrm{O}_{4} / \AA$ & 4.86 & 2.97 & 2.53 & 2.1 & 1.71 & 1.48 \\
$h k l$ & 111 & 220 & 311 & 400 & 422 & 440
\end{tabular}

the amine adduct directly. Incubation of the amine-functionalised MNPs with TNBS solution in $\mathrm{pH} 8$ borate buffer $\left(1 \mathrm{~h}\right.$ at $\left.37{ }^{\circ} \mathrm{C}\right)$, followed by magnetic sedimentation of the particles left unreacted TNBS in solution, which could then be quantified by reaction with valine and the change in absorbance at two wavelengths (340 and $410 \mathrm{~nm}$ ) interpolated into a standard curve. This assay showed that $\sim 1 \mathrm{mmol}$ of reactive amine was present per gram of dry APTESMNP, and that there was little difference between APTES coatings that were formed under either acid catalysed or uncatalysed condensations. This amount of amine on the surface compares well to literature values, with Galeotti et al. finding values from 0.33 to $0.74 \mathrm{mmol} \mathrm{g}^{-1}$ on $20 \mathrm{~nm}$ diameter MNPs using different coating conditions, ${ }^{22}$ whilst Čampelj et al. found values from 0.15 to $0.6 \mathrm{mmol} \mathrm{g}^{-1}$ on $14 \mathrm{~nm}$ diameter MNPs. ${ }^{23}$

\section{Biotinylation of APTES-MNPs}

Biotinylation of APTES-MNPs was performed using 21-(D(+)-biotinylamino)-4,7,10,13,16,19-hexaoxaheneicosanoic acid (Bt-PEG-acid). It was hoped that the inclusion of the PEG spacer might improve MNP binding to avidin by projecting the biotin group further from the MNP surface. The Bt-PEG-acid was activated by pre-incubation with $N, N, N^{\prime}, N^{\prime}$-tetramethyl-O- $(1 H$ benzotriazol-1-yl)uronium hexafluorophosphate (HBTU) and diisopropylethylamine (DIPEA) in dry DMF. This activated 
mixture was added to a suspension of APTES-MNPs in dry DMF, prepared using probe sonication. After sonication of the resulting suspension for a further 30 minutes the sample was shaken overnight in a sealed tube, followed by purification by six magnetic sedimentation-resuspension cycles and removal of the solvent under reduced pressure.

FTIR spectra were obtained for MNPs in dry powder form at each stage of the coating procedure (details in the ESI $\dagger$ ). The spectra obtained were compared with the spectra of APTES-coated $\mathrm{Fe}_{3} \mathrm{O}_{4}$ MNPs reported by Yamaura et al. ${ }^{17 a}$ The Fe-O-Si bond overlaps with the Fe-O stretching of bulk magnetite, but the APTES coating was indicated by a silica stretch at $1117 \mathrm{~cm}^{-1}$ along with methylene $\mathrm{C}-\mathrm{H}$ stretches at $2930 \mathrm{~cm}^{-1}$. The successful addition of the Bt-PEG-acid was difficult to ascertain, due in part to the very low mass ratio of this component, but a new absorption at $1295 \mathrm{~cm}^{-1}$ is consistent with the upper end of the $\mathrm{C}-\mathrm{O}$ stretching region, and a shoulder at $1690 \mathrm{~cm}^{-1}$ is consistent with a secondary amide carbonyl in the solid state. ${ }^{24}$

The efficiency of biotinylation was quantified using the colorimetric 2-(4-hydroxyphenylazo)benzoic acid (HABA) biotin assay, using a modification of the procedure of Lackey et al. ${ }^{25}$ The HABA dye $\left(\lambda_{\max }=350 \mathrm{~nm}, \varepsilon_{350}=20500 \mathrm{M}^{-1} \mathrm{~cm}^{-1}\right)$ binds to the biotin-binding site of avidin to give a strongly coloured complex $\left(\lambda_{\max }=500 \mathrm{~nm}, \varepsilon_{500}=35500 \mathrm{M}^{-1} \mathrm{~cm}^{-1}\right) .{ }^{26} \mathrm{HABA}$ is readily displaced from avidin by biotin $\left(K_{\mathrm{d}(\text { HABA-avidin })}=6 \times 10^{-6} \mathrm{M}, K_{\mathrm{d}(\text { biotin-avidin })}=\right.$ $\left.10^{-13}-10^{-15} \mathrm{M}\right){ }^{26}$ so monitoring the decrease in absorbance at $500 \mathrm{~nm}$ therefore gives a quantitative measure of available biotin.

A HABA: avidin tetramer solution was prepared (4:1 stoichiometry) and the absorbance spectrum recorded. An aliquot of biotinylated MNP suspension of known concentration was added and the sample was incubated for $\sim 300 \mathrm{~s}$ to allow equilibration. The Bt-APTES-MNPs were then magnetically sedimented ( $\sim 1200 \mathrm{~s}$ on a $6000 \mathrm{G} \mathrm{NdFeB}$ magnet) and the spectrum re-measured. Each Bt-APTES-MNPs aliquot gave the expected decrease in absorbance at $500 \mathrm{~nm}$ and increase at $350 \mathrm{~nm}$, with an isosbestic point observed at $407 \mathrm{~nm}$. The decrease in absorbance at $500 \mathrm{~nm}$ was used to calculate the amount of complexed HABA displaced by biotin moieties on the particle surfaces (details in the ESI $\dagger$ ). The Bt-APTES-MNPs prepared using either uncatalysed or acid catalysed APTES deposition procedures were found to have similar quantities of available biotin per gram of MNP, at $13 \pm 5 \mu \mathrm{mol} \mathrm{g}^{-1}$ and $13 \pm 3 \mu \mathrm{mol} \mathrm{g}^{-1}$ respectively. This is consistent with the TNBS assays that had indicated similar levels of available amine resulted from either uncatalysed or acid-catalysed APTES deposition. Given the absence of a difference between these coating methodologies, the uncatalysed APTES coating was then taken forward as the standard technique for nanoparticle functionalisation. Although the HABA:avidin assay at first glance suggested only $2-3 \%$ of surface amines were biotinylated, the size of the avidin tetramer $(50 \times 50 \times 40 \AA)^{26 c}$ introduces a steric barrier that prevents access to all surface biotin groups. By assuming spherical MNPs of radius 30 to $50 \mathrm{~nm}$ and assuming every binding site on the avidin tetramer can be filled, an expected upper limit of 8 to $14 \mu \mathrm{mol} \mathrm{g}^{-1}$ can be calculated, close to the value observed. Although this HABA assay provides only the number of biotin moieties available to avidin rather than the total extent of biotinylation, it shows that the MNPs can be surface saturated with avidin.

\section{Quartz crystal microgravimetry with dissipation (QCM-D) studies}

QCM-D is a technique that relies upon the piezoelectric oscillation of quartz crystals in response to an applied voltage. Monitoring the frequency $(f)$ of these oscillations enables the highly accurate determination of the mass of material deposited on the crystal surface due to the dependence of the oscillation frequency on the total mass of the crystal and any deposited material. Dissipation $(D)$ is a measure of the rate of decay of those oscillations when the voltage is no longer applied. This decay is related to how rapidly the energy of the oscillations is transferred from the crystal to the environment, which is in turn related to the viscoelasticity of any deposited material - a more viscoelastic mass disperses the energy of the oscillations more efficiently, leading to an increase in $D$. The nanogram sensitivity of QCM-D means that a monolayer of proteins or lipids on the sensor surface is well within the analytical range, while monitoring the change in viscoelasticity associated with a mass deposition can indicate the structure of the deposited biomolecule. It has been extensively used to characterise the interactions of large biomolecules, self-assembled particles, nanoparticles and cells with surfaces, ${ }^{27}$ with recent examples showing nanoparticle and vesicle immobilisation can be studied by QCM, e.g. layer-by-layer deposition of Cowpea mosaic viruses, ${ }^{28} \beta$-cyclodextrin vesicles ${ }^{29}$ and gold nanoparticles. ${ }^{30}$ Both biotin-(strept)avidin interactions and saccharide-lectin interactions have been used to link vesicles to a QCM chip, which can be either directly coated with protein ${ }^{31,32}$ or coated via protein recognition of a functionalised phospholipid bilayer on the surface. ${ }^{28}$

QCM-D should therefore be an ideal technique to assess if each of the proposed binding steps - biotinylated vesicles binding to avidin and biotinylated MNPs binding to immobilised avidin can occur and may indicate the relative strength of these binding events. In order to model the vesicle surface, a biotinylated phospholipid bilayer was deposited onto a silica-coated sensor. Biotinylated dipalmitoyl phosphatidylethanolamine with a caproyl spacer between the lipid headgroup and the biotin (Bt-caproyl-PE) was used to dope DOPC vesicles with biotin, with the caproyl spacer giving more flexibility and reach than the biotin lipid used previously. ${ }^{15}$ Flowing a sonicated suspension of DOPC vesicles $\left(0.1 \mathrm{mg} \mathrm{mL}{ }^{-1}\right)$ doped with $0.2 \mathrm{~mol} \%$ Bt-caproyl-PE across the QCM-D chip gave a characteristic 'burst effect' (a, Fig. 3) that indicated a lipid layer was deposited on the sensor surface; the 'burst effect' is caused by the vesicles first settling onto the silica chip then lysing to give a surface bilayer. ${ }^{33}$ Avidin solution $\left(0.1 \mathrm{mg} \mathrm{mL}{ }^{-1}\right)$ was then flowed over the deposited bilayer, producing a decrease in frequency $(f)$ and increase in dissipation $(D)$ (b, Fig. 3) that indicated deposited mass and increased viscoelastic character. ${ }^{34} \mathrm{~A}$ non-biotinylated DOPC bilayer on the chip gave only a small change in $f$ and $D$ upon addition of avidin solution, suggesting specific biotin-avidin binding between the Bt-caproyl-PE doped bilayer and avidin was responsible for this increase in mass (details in the ESI $\dagger$ ). 


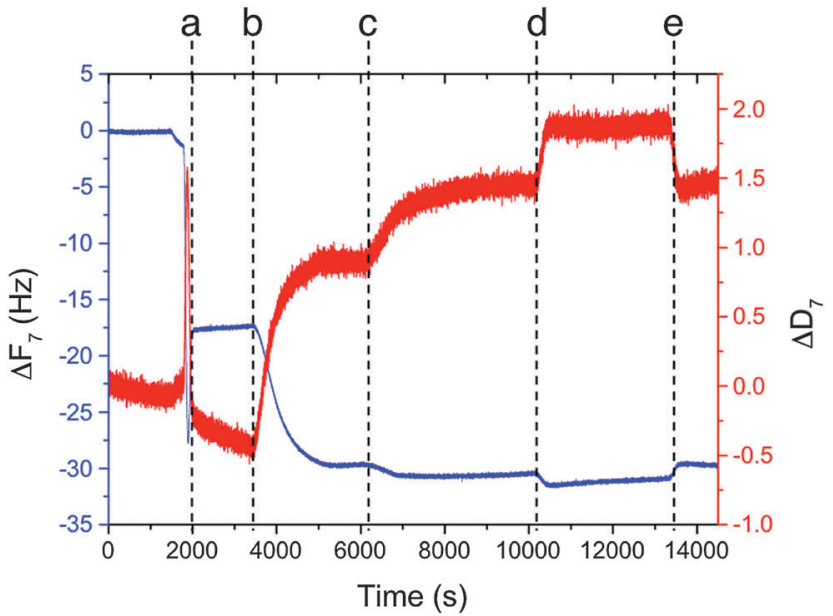

Fig. 3 Plot of frequency and dissipation change over time, calculated from the frequency values of the seventh overtone. Marked timepoints indicate when each component reached the sensor: (a) Bt-DOPC vesicles - 'burst effect', (b) avidin, (c) Bt-APTES-MNPs, (d) biotin, (e) 'blank' buffer

Flowing a solution of Bt-APTES-MNPs over the resulting avidincoated biotinylated bilayer resulted in a further increase in deposited mass and increased viscoelastic character (c, Fig. 3). Flowing uncoated MNPs or APTES-MNPs over an avidin-coated biotinylated bilayer gave a much smaller response than the biotinylated particles, with uncoated MNPs causing a slight decrease in deposited mass (details in the ESI $\dagger$ ), both observations strongly supporting the involvement of specific biotinavidin binding events.

A solution of biotin $\left(0.1 \mathrm{mg} \mathrm{mL^{-1 }}\right)$ was then flowed over the sensor (d, Fig. 3) to see if competitive binding by free biotin could desorb bound material. Instead, a further increase in deposited mass and viscoelasticity was observed. This change seems to be due to non-specific association of biotin with the lipid bilayer, as flowing buffer over the sensor chip reversed the increase in mass, with $f$ and $D$ rapidly returning to their prior values (e, Fig. 3). Interestingly, these data correlated with optical observations of MNPV suspensions that had indicated that aggregation was not reversible upon free biotin addition.

\section{Magnetic nanoparticle-vesicle aggregate (MNPV) formation}

Prior to the formation of MNPVs, the MNPs were saturated with a layer of bound avidin. Bt-APTES-MNPs $\left(0.5 \mathrm{mg} \mathrm{mL}{ }^{-1}, 2 \mathrm{~mL}\right)$ and avidin ( $50 \mu \mathrm{L}$ of $10 \mathrm{mg} \mathrm{mL}^{-1}$ stock) were incubated together in PBS buffer at $37{ }^{\circ} \mathrm{C}$ for $\sim 300 \mathrm{~s}$; at this $0.55: 1$ ratio of avidin: biotin, sufficient avidin binding sites are present to bind to more than $50 \%$ of the biotin available on the MNPs, suppressing avidin-mediated crosslinking of nanoparticles. The avidin-coated MNPs were then added to a suspension of biotinylated DPPC vesicles (formed by extrusion through $800 \mathrm{~nm}$ diameter polycarbonate membranes, $20 \mathrm{mM}$ lipid, 0.2\% Bt-caproyl$\mathrm{PE}$ ), and the mixture incubated together for 40 minutes at $37{ }^{\circ} \mathrm{C}$ to form the MNPVs ( $2 \mathrm{~mL}$, final [lipid] $2 \mathrm{mM}, 0.5 \mathrm{mg} \mathrm{mL}^{-1} \mathrm{MNP}$ ). Successful formation of MNPVs was indicated by the formation of a homogenous brown mass that was responsive to external magnetic fields, with few non-aggregated vesicles in suspension. Fluorescence microscopy on this magnetic material confirmed aggregation, showing MNPVs of diameters from 5 to $15 \mu \mathrm{m}$ (see ESI $\dagger$ ). Magnetic sedimentation could then be used to remove non-magnetic material.

\section{Release of 5/6-CF from MNPVs}

In our previous release experiments with MNPVs, these nanocarriers had been embedded in a hydrogel matrix, ${ }^{15}$ but release from MNPVs in suspension had not been investigated. The release of encapsulated compounds from MNPVs is believed to depend upon the MNP-mediated melting of the vesicle bilayers at the bilayer melting temperature $\left(T_{\mathrm{m}}\right)$, which for DPPC vesicles is $41{ }^{\circ} \mathrm{C}$. The large increase in membrane permeability and rapid escape of the vesicle contents is due to disorder at boundaries between coexisting solid ordered and liquid ordered phases ${ }^{6}$ which allows the passage of water-soluble compounds through the bilayer.

The magnetically-triggered release of vesicle contents from MNPVs in suspension was initially quantified using the selfquenching fluorescent dye 5/6-carboxyfluorescein (5/6-CF). Vesicles containing encapsulated 5/6-CF $(50 \mathrm{mM})$ were crosslinked by avidin-coated MNPs, and the resulting MNPVs incubated at $37{ }^{\circ} \mathrm{C}$. Once formed, these MNPVs had good contents retention, with $<20 \%$ leakage of 5/6-CF after $24 \mathrm{~h}$ at $37^{\circ} \mathrm{C}$ (Fig. 4). Release was triggered by exposing samples to an AMF pulse $(392 \mathrm{kHz})$ for $300 \mathrm{~s}$, a duration that SAR data for the MNPs suggested would be sufficient to heat the MNPV bilayers beyond their $T_{\mathrm{m}}$ (details in ESI $\dagger$ ). Release of 5/6-CF was quantified by measuring the fluorescence $\left(\lambda_{\mathrm{ex}}=492 \mathrm{~nm}, \lambda_{\mathrm{em}}=517 \mathrm{~nm}\right)$ of the supernatant buffer. Samples of MNPVs incubated at physiological temperature $\left(37{ }^{\circ} \mathrm{C}\right)$ but without AMF exposure served as a control to assess the effect of the AMF, whilst samples incubated at $42{ }^{\circ} \mathrm{C}$ (above the $T_{\mathrm{m}}$ of DPPC) should provide an upper limit for the amount of release expected. The fluorescence recovery in each case was converted to percentage release by using the fluorescence obtained after incubation at $42{ }^{\circ} \mathrm{C}$ for $15 \mathrm{~h}$ and brief sonication as the maximum value (Fig. 4).

A seven-fold increase in 5/6-CF in the supernatant occurred immediately after exposure to an AMF, comprising around 30\%

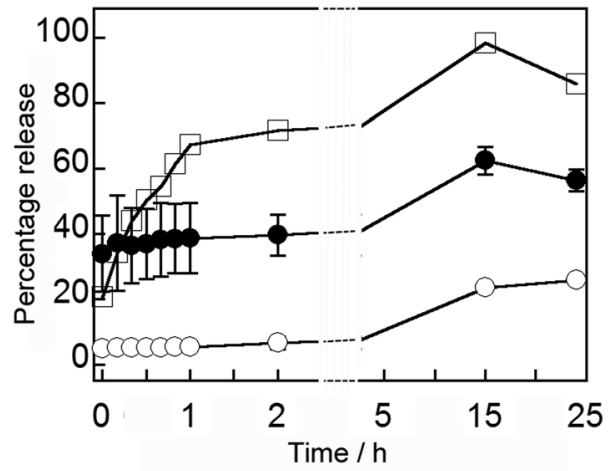

Fig. 4 Average percentage release over $24 \mathrm{~h}$ of 5/6-CF from MNPVs: incubated at $42{ }^{\circ} \mathrm{C}(\square)$; incubated at $37^{\circ} \mathrm{C}$ no $A M F(O$, two repeats); incubated at $37^{\circ} \mathrm{C}$ after $\operatorname{AMF}(\bullet$, three repeats). 
of encapsulated material (with $100 \%$ release corresponding to $\sim 5 \mu \mathrm{M} 5 / 6$-CF in the supernatant), and this $\sim 30 \%$ difference was maintained over the next $24 \mathrm{~h}$ (Fig. 4). Given the steady increase observed for the MNPV sample incubated at $42{ }^{\circ} \mathrm{C}$, this observation suggests that a discrete release event occurs during AMF exposure, with no increase in membrane permeability after the AMF is removed. Release was slower than previous release experiments with MNPVs embedded in a hydrogel matrix, ${ }^{15}$ suggesting that perhaps the hydrogel enhances localised heating and/or the gel fibrils are transmitting mechanical shear forces produced by rapidly oscillating particles to the trapped vesicles.

Initial experiments with MNPVs prepared using different methodology ${ }^{35}$ had indicated that although both MNPs and AMF exposure were required to give release, the avidin coating on nanoparticles did not give a significant improvement in release at $37{ }^{\circ} \mathrm{C}$. This correlates with observations from the QCM-D assays that biotin did not reverse the adhesion of MNPs to avidincoated bilayers, and also implied that biotinylated APTES-MNPS may associate with phospholipid bilayers in the absence of specific interactions. The zeta potential of Bt-APTES-MNPs was $+8 \mathrm{mV},{ }^{36}$ perhaps due to unreacted amines, and may help the interaction with vesicles doped with anionic Bt-caproyl-PE; the MNPVs produced have a net zeta potential of $+4 \mathrm{mV}$. Alternatively the biotin layer may give increase in hydrophobic character to the MNPs.

Membrane melting at the $T_{\mathrm{m}}$ has been proposed to be the release mechanism, but this had not been separately investigated. Fluorescence microscopy showed that the multi-compartment structure of the MNPVs remained intact after the AMF pulse (see the ESI $\dagger$ ), fitting our model that release occurs after the formation of transient pores at the intersection between phases in the membrane. Subsequent sonication could dissociate the aggregates. To investigate the release mechanism, a set of comparative experiments were carried out using DOPC MNPVs in the place of DPPC MNPVs. DOPC bilayers are already in a liquid crystalline phase at room temperature $\left(T_{\mathrm{m}}-17{ }^{\circ} \mathrm{C}\right),{ }^{37}$ and will not undergo a phase transition in response to heating that gives a concomitant release event. Parallel DPPC and DOPC MNPV samples with encapsulated 5/6-CF were exposed to an AMF pulse (300 s) at $37{ }^{\circ} \mathrm{C}$, and the fluorescence recovery measured after 45 minutes. These studies showed no 5/6-CF release from DOPC MNPVs above the background leakage rate; this was $c a$. a third the amount of release from the DPPC MNPV sample exposed to an AMF (see the $\mathrm{ESI}^{\dagger}$ ). This supports a release mechanism based upon magnetically induced heating of the MNPs, rather than MNPs moving under magnetophoretic force to shear or mechanically weaken the vesicle membranes.

\section{Release of cytochrome $\boldsymbol{c}$ from MNPVs}

Having established conditions for the controlled release of a small molecule (0.4 $\mathrm{kDa}$ for 5/6-CF) from these redesigned MNPVs, small proteins were identified as a key class of biomolecule for controlled release. Cytochrome $c$ was identified as an attractive test case as it is relatively small in size $(\sim 12 \mathrm{kDa})$ and possesses a highly coloured heme prosthetic group $\left(\varepsilon_{410}=\right.$ $\left.106000 \mathrm{M}^{-1} \mathrm{~cm}^{-1}\right){ }^{38}$
Encapsulation of proteins and enzymes presents a number of challenges, for example many proteins interact strongly with lipid membranes, particularly membranes containing charged lipids; charge dependent "encapsulation", which may in fact be electrostatic association with the bilayer, has been noted. ${ }^{39}$ Additionally, the large size of some proteins can be detrimental to their encapsulation efficiency and release rates. Lipid film rehydration and extrusion techniques are recognised as being one of the most effective methods for efficient encapsulation of proteins in LUVs, ${ }^{40}$ but a number of studies have indicated better protein encapsulation by including multiple freeze-thaw cycles during vesicle formation. This increase in efficiency has been rationalised as the rupture of vesicles during the freezethaw process, enabling equilibration with the surrounding medium and inducing more uniform unilamellar vesicles with greater entrapped volumes. ${ }^{41-43}$ To find the optimum balance between improving encapsulation and enzyme denaturation, Colletier et al. studied the effect of freeze-thaw cycles on acetylcholine esterase, finding that 10 freeze-thaw cycles were most effective. ${ }^{41}$

Biotinylated DPPC vesicles (20 mM lipid) containing cytochrome $c$ (10 $\mathrm{mg} \mathrm{mL}^{-1}$ in PBS) were therefore prepared using this freeze-thaw methodology. Gel permeation chromatography (GPC) was ineffective for separating unencapsulated protein due to the size exclusion limit of the gel used. Instead multiple cycles of centrifugation $(2200 \mathrm{~g}, 15$ minutes), removal and replacement of the supernatant were used until no absorbance at $410 \mathrm{~nm}$ could be detected above the baseline.

MNPVs were then prepared by crosslinking these biotinylated vesicles with avidin-coated biotinylated APTES-MNPs. Exposure to an AMF pulse (300 s) at $37{ }^{\circ} \mathrm{C}$ gave a significant increase in the amount of cytochrome $c$ in the supernatant compared with samples that were only incubated at $37{ }^{\circ} \mathrm{C} ; \sim 65 \%$ versus $\sim 15 \%$ respectively (Fig. 5). Protein release largely occurred soon after AMF exposure, with little subsequent leakage. Interestingly, if the freeze-thaw cycle were omitted during vesicle preparation then no cytochrome $c$ release was observed, suggesting little encapsulation. Although cytochrome $c$ is not catalytically active, the ability of this MNPV formulation to release proteins suggested that enzymes should also be deliverable.

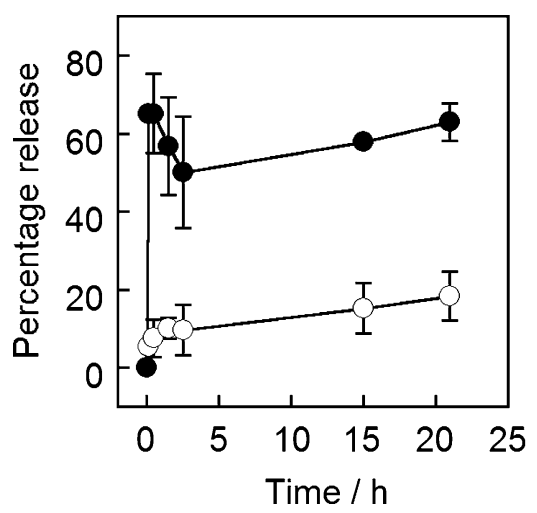

Fig. 5 Release of cytochrome $c$ from MNPVs as a percentage of maximum release (a sample incubated at $42^{\circ} \mathrm{C}$ and sonicated). (O) No AMF, (O) AMF. 


\section{Release of FITC-labelled trypsin from MNPVs}

The commonly used protease trypsin $(23 \mathrm{kDa})$ was chosen to assess if an enzyme could be encapsulated and released from MNPVs whilst retaining activity. To track the release of the enzyme, trypsin was fluorescently labelled using fluorescein isothiocyanate (FITC), which allowed released protein to be detected in the supernatant following removal of the MNPVs. Vesicles entrapping FITC-trypsin $\left(10 \mathrm{mg} \mathrm{mL}^{-1}\right)$ were prepared using the modified freeze-thaw technique, and purified by sedimentation as used for the cytochrome $c$ vesicles. ${ }^{41,43}$ MNPVs were then prepared as per the method employed previously.

Aliquots of MNPVs suspension $(200 \mu \mathrm{L})$ were transferred to centrifuge tubes containing PBS $(1 \mathrm{~mL})$. If required, they were exposed to an AMF pulse (300 s) followed by centrifugation $(2200 \times g, 10$ minutes $)$ to sediment the suspended MNPVs. The fluorescence emission of the samples at $525 \mathrm{~nm}\left(\lambda_{\mathrm{ex}}=495 \mathrm{~nm}\right)$ was then recorded at each time point. All samples were incubated at $37{ }^{\circ} \mathrm{C}$ and protected from light between measurements. The fluorescence of the supernatant from AMF exposed samples and non-AMF exposed controls was monitored for $15 \mathrm{~h}$, and the fluorescent intensities interpolated into a standard curve (see the ESI $\dagger$ ) to give the concentration of released FITC-trypsin in the supernatant (Fig. 6).

As with 5/6-CF and cytochrome $c$ release, a clear release event occurred during the AMF pulse, that released $\sim 0.3 \mu \mathrm{g} \mathrm{mL} \mathrm{mL}^{-1}$ of trypsin. This was followed by release comparable to rates of leakage from both samples (with AMF, no AMF), to give $\sim 0.6 \mu \mathrm{g} \mathrm{mL} \mathrm{m}^{-1}$ trypsin in the magnetically triggered sample after $15 \mathrm{~h}$, which is somewhat lower than the concentration measured for cytochrome $c$ $\left(2.7 \mu \mathrm{g} \mathrm{mL}{ }^{-1}\right) .{ }^{44}$ Nonetheless the successful release of trypsin and cytochrome $c$, as well as hyaluronidase, ${ }^{44}$ indicate that the MNPV system is able to effectively encapsulate and release proteins of biological relevance.

\section{Trypsin activity assay}

To determine if magnetically released trypsin retained its activity, a protease activity assay was performed on the supernatant of trypsinloaded MNPV samples. A commercially available kit was used (Fluorescent Protease Activity Assay, PF0100, Sigma-Aldrich UK)

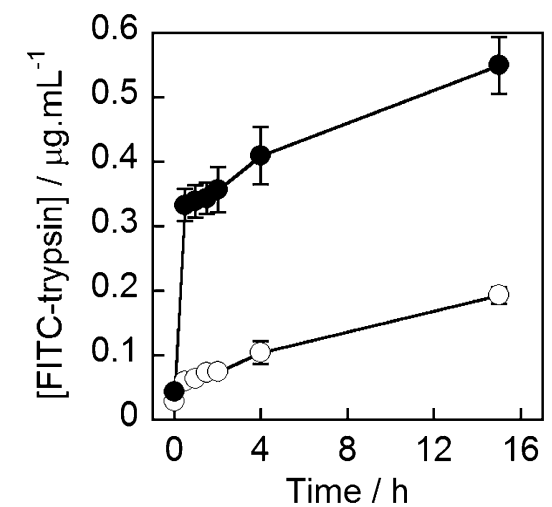

Fig. 6 Concentration of FITC-trypsin in the buffer supernatant of MNPV samples (O) not exposed to AMF, (O) AMF exposed. AMF pulse completed at $t=5$ minutes. and the assay performed as described by Cupp-Enyard et $a .^{45}$ This assay uses FITC-labelled casein as a substrate for protease activity, which is incubated with the trypsin containing sample for $1 \mathrm{~h}$. After this time residual protein is precipitated by trichloroacetic acid addition, and removed by centrifugation to leave only fluorescent protein fragments in solution. The concentration of soluble fragments is proportional to the protease activity of the sample and the fluorescence of the supernatant can therefore be used to quantify protease activity. Activity can be calculated from the fluorescence data by reference to a standard curve established using aliquots of trypsin with known activity (in BAEE units).

MNPVs (2 mM lipid, $2 \mathrm{~mL}$ ) containing unlabelled trypsin (from lyophilised trypsin-EDTA solution, $10 \mathrm{mg} \mathrm{mL}^{-1},<7500$ BAEE units $\left.\mathrm{mL}^{-1}\right)^{46}$ were prepared and treated in an identical fashion to those containing FITC-trypsin. After incubation or exposure to an AMF (300 s), the assay was performed on aliquots $(10 \mu \mathrm{L})$ of the supernatant after centrifugation, which was added to FITC-casein solution ( $20 \mu \mathrm{L}$ of supplied reagent) and buffer $(20 \mu \mathrm{L})$. The supernatant from MNPVs exposed to an AMF gave a clear response in this assay compared to that from MNPVs that were not exposed to an AMF (Fig. 7). The trypsin activity in samples exposed to an AMF correlates well with the FITC-trypsin release experiments, although the difference in activity between AMF and no AMF samples was somewhat less than what might be expected based on the relative quantities of FITC-trypsin released. Trypsin activity in the samples may decrease over time due to 'self-digestion' of trypsin at high concentrations (e.g. encapsulated concentrations) and over long incubation times. Despite this possible loss of activity, the results still clearly demonstrate magnetically triggered release of active enzymes is achievable in the MNPV system.

Trypsin activity can also be analysed using the chromogenic substrate $N$-benzoyl-L-arginine 4-nitroanilide hydrochloride (BAPNA), which after digestion gives the nitroaniline chromophore. ${ }^{47}$ This polar and cationic amino acid derivative seemed to be the ideal substrate to assess if two components of a simple chemical network (enzyme and substrate) could be encapsulated in separate vesicle compartments and released together or sequentially.

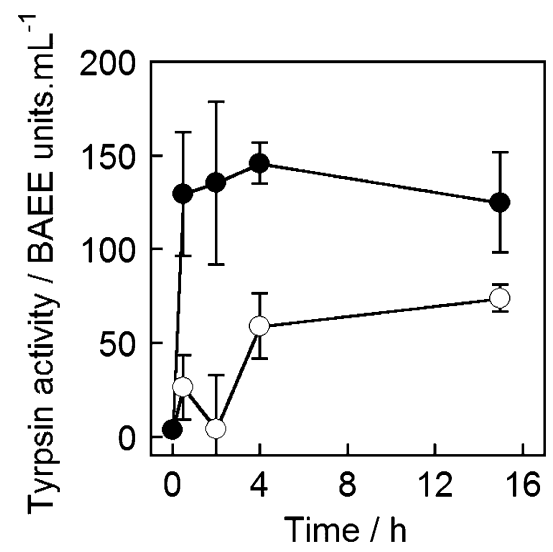

Fig. 7 Trypsin activity in the supernatant of MNPV samples that were: exposed to AMF and (O) not exposed to AMF. Average of four repeats. 
However these experiments revealed some of the limitations of encapsulation technology. The BAPNA substrate was not well encapsulated, with only a low level of material released after thermal treatment of DPPC vesicles. Furthermore, these BAPNAcontaining biotinylated vesicles could not be aggregated by avidin-coated biotinylated MNPs. Measurements of the zeta potential and size of these BAPNA containing vesicles showed they had a zeta potential of $+6 \mathrm{mV}$ and an average diameter of $260 \mathrm{~nm}$, smaller than expected size of $800 \mathrm{~nm}$. Given that both Bt-APTES-MNPs and avidin are positively charged, ${ }^{26 c}$ this positive surface charge on the vesicles may have inhibited crosslinking by avidin-coated Bt-APTES-MNPs.

\section{Conclusions}

Adhesive magnetic nanoparticles have been shown to be a useful addition to the suite of tools available to mediate the transit of compounds across phospholipid bilayers. Careful modification of the design of a composite nanomaterial containing phospholipid vesicle compartments crosslinked by avidin-coated MNPs has allowed the release of small molecules, proteins and enzymes in solution in response to an alternating magnetic field (AMF).

One key modification was the surface functionalization of MNPs using an APTES coating followed by biotinylation, which has proven to be a reproducible method for generating adhesive magnetic nanoparticles that display biotin. This methodology allows each step of the MNP functionalisation process to be quantified using TNBS or HABA assays respectively. The HABA/avidin assay indicated that sufficient biotin could be grafted onto APTES-MNPs to produce near saturation of avidin on the surface of the MNPs.

QCM-D showed that these biotinylated APTES-MNPs were able to bind to avidin immobilised on a DOPC bilayer doped with $0.2 \mathrm{~mol} \%$ Bt-caproyl-PE. The QCM-D data also showed that this interaction was not reversible upon the addition of free biotin, suggesting that additional interactions may aid adhesion of the biotinylated APTES-MNPs to bilayer, perhaps due to the hydrophobic biotin layer causing some ingress of the MNPs into the vesicle surface. Indeed QCM-D showed no interaction between avidin-coated Bt-DOPC bilayers and the uncoated MNPs or APTES-MNPs.

The QCM-D studies were consistent with the successful formation of magnetic nanoparticle-vesicle aggregates upon mixing of avidin-coated biotinylated APTES-MNPs and DPPC vesicles doped with $0.2 \mathrm{~mol} \%$ Bt-caproyl-PE. A $300 \mathrm{~s}$ magnetic pulse could then release small molecules, proteins and enzymes from these new MNPVs in suspension. Studies of the magnetic release of the dye 5/6-CF combined with the QCM-D measurements suggest that surface charge and nanoparticle hydrophobicity may play a key role in maintaining the interaction between the vesicle membranes and MNPs.

The release of biomolecules like cytochrome $c$ and trypsin, and the demonstration that catalytic activity is retained by the latter, shows that these constructs should have an exciting future for biomimetic compartmentalised networks as well as for the remote delivery of biopharmaceuticals in vivo. However, a number of barriers to progress in this area still exist. Encapsulation technology for LUVs is a problem as specific conditions are often required for the entrapment of large biomolecules, and the quantity of entrapped biomolecule can be low. Although small hydrophilic compounds are easier to encapsulate, not all reagents are suitable, especially if they have low solubility, significant hydrophobic character or groups, like guanidinium cations, that interact with phospholipid bilayers. Cost is also a significant issue for both large biomolecules and some smaller compounds, unless an efficient method for recycling non-encapsulated materials is used. However new hydration techniques that allow bulk production of GUVs encapsulating a larger range of biomolecules and substrates may allow GUV self-assembly to be used in the place of LUV selfassembly. ${ }^{48}$ Further efficiencies could be gained by copying natural systems like gap junctions more closely, e.g. combining selfassembly, adhesion and the ability to mediate contents transfer from vesicle compartment to vesicle compartment. Investigations into such biomimetic systems are ongoing.

\section{Acknowledgements}

We thank Mr Lan Nguyen for TEM images of MNPs and Biolin Scientific (UK) for the generous loan of a Q-Sense E4 instrument. $\mathrm{AB}$ would like to thank the BBSRC for a PhD studentship and ICP would like to acknowledge financial support from a FP7 Marie Curie Intra-European Fellowship (MagNanoVes, grant 328025).

\section{Notes and references}

1 G. S. Goldberg, V. Valiunas and P. R. Brink, Biochim. Biophys. Acta, 2004, 1662, 96-101.

2 P. A. Beales and T. K. Vanderlick, Adv. Colloid Interface Sci., 2014, 207, 290-305.

3 G. Villar, A. D. Graham and H. Bayley, Science, 2013, 340, 48-52.

4 Y. Elani, R. V. Law and O. Ces, Nat. Commun., 2014, 5, DOI: 10.1038/ncomms6305.

5 A. Karlsson, R. Karlsson, M. Karlsson, A.-S. Cans, A. Strömberg, F. Ryttsén and O. Orwar, Nature, 2001, 409, 150-152.

6 S. J. Webb, Acc. Chem. Res., 2013, 46, 2878-2887.

7 S. J. Webb, L. Trembleau, R. J. Mart and X. Wang, Org. Biomol. Chem., 2005, 3, 3615-3617.

8 S. Chiruvolu, S. Walker, J. Israelachvili, F. J. Schmitt, D. Leckband and J. A. Zasadzinski, Science, 1994, 264, 1753-1756.

9 (a) B. van Lengerich, R. J. Rawle and S. G. Boxer, Langmuir, 2010, 26, 8666-8672; (b) C. Yoshina-Ishii and S. G. Boxer, J. Am. Chem. Soc., 2003, 125, 3696-3697.

10 (a) M. Ma and D. Bong, Acc. Chem. Res., 2013, 46, 2988-2997; (b) J. Voskuhl, C. Wendeln, F. Versluis, E.-C. Fritz, O. Roling, H. Zope, C. Schulz, S. Rinnen, H. F. Arlinghaus, B. J. Ravoo and A. Kros, Angew. Chem., Int. Ed., 2012, 51, 12616-12620.

11 C. M. Paleos, Z. Sideratou and D. Tsiourvas, ChemBioChem, 2001, 2, 305-310. 
12 R. J. Mart, K. P. Liem, X. Wang and S. J. Webb, J. Am. Chem. Soc., 2006, 128, 14462-14463.

13 R. J. Mart, K. P. Liem and S. J. Webb, Pharm. Res., 2009, 26, 1701-1710.

14 K. P. Liem, R. J. Mart and S. J. Webb, J. Am. Chem. Soc., 2007, 129, 12080-12081.

15 (a) F. de Cogan, A. Booth, J. E. Gough and S. J. Webb, Soft Matter, 2013, 9, 2245-2253; (b) F. de Cogan, A. Booth, J. E. Gough and S. J. Webb, Angew. Chem., Int. Ed., 2011, 50, 12290-12293.

16 (a) Y. Chen, Y. Chen, D. Xiao, A. Bose, R. Deng and G. D. Bothun, Colloids Surf., B, 2014, 116, 452-458; (b) V. Plassat, C. Wilhelm, V. Marsaud, C. Ménager, F. Gazeau, J.-M. Renoir and S. Lesieur, Adv. Funct. Mater., 2011, 21, 83-92; (c) M. Babincová, P. Čičmanec, V. Altanerová, Č. Altaner and P. Babinec, Bioelectrochemistry, 2002, 55, 17-19.

17 (a) M. Yamaura, R. L. Camilo, L. C. Sampaio, M. A. Macedo, M. Nakamura and H. E. Toma, J. Magn. Magn. Mater., 2004, 279, 210-217; (b) I. J. Bruce and T. Sen, Langmuir, 2005, 21, 7029-7035; (c) A. del Campo, T. Sen, J.-P. Lellouche and I. J. Bruce, J. Magn. Magn. Mater., 2005, 293, 33-40; (d) I. Koh, X. Wang, B. Varughese, L. Isaacs, S. H. Ehrman and D. S. English, J. Phys. Chem. B, 2006, 110, 1553-1558; (e) X.-C. Shen, X.-Z. Fang, Y.-H. Zhou and H. Liang, Chem. Lett., 2004, 33, 1468-1469.

18 T. Coxon, PhD thesis, University of Manchester, 2015.

19 S. Sun, H. Zeng, D. B. Robinson, S. Raoux, P. M. Rice, S. X. Wang and G. Li, J. Am. Chem. Soc., 2004, 126, 273-279.

20 K. Satake, T. Okuyama, M. Ohashi and T. Shinoda, J. Biochem., 1960, 47, 654-660.

21 F. Edwards-Lévy, M.-C. Andry and M.-C. Lévy, Int. J. Pharm., 1993, 96, 85-90.

22 F. Galeotti, F. Bertini, G. Scavia and A. Bolognesi, J. Colloid Interface Sci., 2011, 360, 540-547.

23 S. Čampelj, D. Makovec and M. Drofenik, J. Magn. Magn. Mater., 2009, 321, 1346-1350.

24 D. H. Williams and I. Fleming, Spectroscopic Methods in Organic Chemistry, McGraw Hill, Maidenhead, 5th edn, 1995.

25 C. A. Lackey, N. Murthy, O. W. Press, D. A. Tirrell, A. S. Hoffman and P. S. Stayton, Bioconjugate Chem., 1999, 10, 401-405.

26 (a) N. M. Green, in Methods Enzymol., ed. D. B. McCormick and L. D. Wright, Academic Press, New York, 1970, vol. 18, Part A, pp. 418-424; (b) K. P. Liem, G. T. Noble, S. L. Flitsch and S. J. Webb, Faraday Discuss., 2010, 145, 219-233; (c) H. Orelma, L. Johansson, I. Filpponen, O. J. Rojas and J. Laine, Biomacromolecules, 2012, 13, 2802-2810.

27 (a) R. E. Speight and M. A. Cooper, J. Mol. Recognit., 2012, 25, 451-473; (b) C. I. Cheng, Y.-P. Chang and Y.-H. Chu, Chem. Soc. Rev., 2012, 41, 1947-1971.

28 N. F. Steinmetz, E. Bock, R. P. Richter, J. P. Spatz, G. P. Lomonossoff and D. J. Evans, Biomacromolecules, 2008, 9, 456-462.

29 O. Roling, C. Wendeln, U. Kauscher, P. Seelheim, H.-J. Galla and B. J. Ravoo, Langmuir, 2013, 29, 10174-10182.
30 E. Mahon, Z. Mouline, M. Silion, A. Gilles, M. Pinteala and M. Barboiu, Chem. Commun., 2013, 49, 3004-3006.

31 D. Thakar, E. Migliorini, L. Coche-Guerente, R. Sadir, H. Lortat-Jacob, D. Boturyn, O. Renaudet, P. Labbe and R. P. Richter, Chem. Commun., 2014, 50, 15148-15151.

32 (a) E. Mahon, T. Aastrup and M. Barboiu, Chem. Commun., 2010, 46, 2441-2443; (b) Z. Mouline, E. Mahon, E. Gomez, V. Barragan-Montero, J.-L. Montero and M. Barboiu, Chem. Commun., 2014, 50, 731-733.

33 N.-J. Cho, C. W. Frank, B. Kasemo and F. Höök, Nat. Protoc., 2010, 5, 1096-1106.

34 Doping the bilayers with the same loading of biotinylated DPPE without the caproyl linker give bilayers that were less effective at capturing avidin from solution, giving only $10 \%$ of the change in mass on the QCM-D chip under the same conditions.

35 The MNPVs used in these preliminary studies were created using MNP suspensions prepared by bath sonication rather than probe sonication, and tended to have a higher rate of leakage (20-40\% release after 45 minutes). The corresponding AMF-triggered release values (either with or without avidin) were $50-100 \%$.

36 A zeta potential of $+9 \mathrm{mV}$ is reported for APTES-MNPs in $0.15 \mathrm{M} \mathrm{NaCl}$ at $\mathrm{pH}$ 7. See: R. A. Bini, R. F. C. Marques, F. J. Santos, J. A. Chaker and M. Jafelicci, Jr., J. Magn. Magn. Mater., 2012, 324, 534-539.

37 J. R. Silvius, Thermotropic Phase Transitions of Pure Lipids in Model Membranes and Their Modifications by Membrane Proteins, Lipid-Protein Interactions, John Wiley \& Sons, Inc., New York, 1982.

38 (a) J. Babul and E. Stellwagen, Biochemistry, 1972, 11, 1195-1200; (b) Y.-L. P. Ow, D. R. Green, Z. Hao and T. W. Mak, Nat. Rev. Mol. Cell Biol., 2008, 9, 532-542.

39 (a) M. C. Annesini, L. Di Giorgio, L. Di Marzio, A. FinazziAgrò, A. L. Serafino and G. Mossa, J. Liposome Res., 1993, 3, 639-648; (b) B. Solomon and I. R. Miller, Biochim. Biophys. Acta, Biomembr., 1976, 455, 332-342.

40 P. Walde and S. Ichikawa, Biomol. Eng., 2001, 18, 143-177. 41 J.-P. Colletier, B. Chaize, M. Winterhalter and D. Fournier, BMC Biotechnol., 2002, 2, 9-17.

42 U. Pick, Arch. Biochem. Biophys., 1981, 212, 186-194.

43 C. J. Chapman, W. L. Erdahl, R. W. Taylor and D. R. Pfeiffer, Chem. Phys. Lipids, 1990, 55, 73-83.

44 A. Booth, PhD thesis, University of Manchester, 2015.

45 C. Cupp-Enyard, J. Visualized Exp., 2009, 30, e1514, DOI: 10.3791/1514.

46 From PAA trypsin $(1 \times)$ 0.25\% in PBS. Supplied as 250 USP units per $\mathrm{mg}$ trypsin. One USP unit $=\sim 3$ BAEE units.

47 B. Kassell, Methods Enzymol., 1970, 19, 844-852.

48 (a) N. L. Mora, J. S. Hansen, Y. Gao, A. A. Ronald, R. Kieltyka, N. Malmstadt and A. Kros, Chem. Commun., 2014, 50, 1953-1955; (b) K. S. Horger, D. J. Estes, R. Capone and M. Mayer, J. Am. Chem. Soc., 2009, 131, 1810-1819. 